website extra

Additional information

appears on the

Evidence-Based

Mental Health

website

www.ebmentalhealth.

com

\section{A depression management programme reduced depression in frequent users of healthcare but did not reduce healthcare visits}

Katzelnick DJ, Simon GE, Pearson SD, et al. Randomized trial of a depression management program in high utilizers of medical care. Arch Fam Med 2000 Apr;9:345-51.

\section{QUESTION: Does a programme to identify and treat depression in frequent users of medical care improve clinical outcomes and reduce total healthcare use?}

\section{Design}

Cluster randomised \{allocation concealment*\}†, blinded (telephone assessment)*, controlled trial with 1 year of follow up.

\section{Setting}

Primary care clinics of 3 health management organisations (HMOs) in the US (Wisconsin, Washington, and Massachusetts).

\section{Patients}

407 patients who were 25-63 years of age (mean age $45 \mathrm{y}$, $77 \%$ women) with continuous health plan enrolment for $\geqslant 2$ years, were frequent healthcare users (ambulatory visits per year above the 85th percentile for previous 2 years), and screened positive for current major depression or major depression in partial remission with a score $\geqslant 15$ on the Hamilton Depression Rating Scale (HDRS). 47\% had $\geqslant 1$ chronic medical disorder. Exclusion criteria included recent treatment for substance abuse, previous treatment for schizophrenia or bipolar disorder, life threatening medical illness, or active mental health specialty treatment for depression. 93\% of patients completed the blinded telephone assessment at 12 months. this abstract appears in ACP Journal Club.

A depression management programme $(D M P) v$ usual care for frequent users of general medical care

\begin{tabular}{|c|c|c|c|}
\hline \multirow[b]{2}{*}{ Outcomes } & \multicolumn{2}{|c|}{ Mean score decrease } & \\
\hline & DMP & Usual care & score decrease \\
\hline
\end{tabular}

\begin{tabular}{llll} 
HDRS score at 6 weeks & 3.3 & 2.0 & 1.3 \\
\hline HDRS score at 3 months & 5.6 & 3.9 & 1.7 \\
\hline HDRS score at 6 months & 7.3 & 4.0 & 3.3 \\
\hline HDRS score at 12 months & 9.2 & 5.6 & 3.6
\end{tabular}

fHDRS=Hamilton Depression Rating Scale. Scores corrected for intracluster correlation among patients of the same physician. All differences are statistically significant.

\section{Intervention}

82 physician practices were allocated to the depression management programme (DMP) $(n=218)$, and 81 were allocated to usual care $(\mathrm{n}=189)$. DMP consisted of physician education (2 hour training session and provision of psychiatrists as consultants at each HMO), patient education (a booklet entitled Depression isn't just a medical problem and videotaped educational materials), antidepressant treatment (pharmacotherapy algorithm adjusted for individual clinical need), and treatment coordination.

\section{Main outcome measures}

Change in HDRS scores and number of healthcare visits.

\section{Main results}

Analysis was by intention to treat. Patients in the DMP group had greater improvements in depression score at 6 weeks $(\mathrm{p}=0.04), 3$ months $(\mathrm{p}=0.02), 6$ months $(\mathrm{p}<0.001)$, and 12 months $(\mathrm{p}<0.001)$ than patients in the usual care group (table). Patients in the DMP group had more healthcare visits (mean increase 1.6 visits) during 12 months of follow up relative to the previous year whereas patients in the usual care group had a decrease in healthcare visits (mean decrease 2.0 visits) ( $p=0.02$ for the difference between groups).

\section{Conclusion}

In depressed patients who are frequent users of general medical care, a depression management programme led to greater clinical improvement but did not decrease healthcare visits.

*See glossary.

†Information provided by author.

\title{
COMMENTARY
}

Depressive illness is common, disabling, and mostly managed in primary care without recourse to specialist services. Received wisdom is that management is suboptimal: up to $50 \%$ of cases are missed by practitioners, and treatment of detected cases is of inadequate intensity and duration. Educational interventions for practitioners using clinical guidelines to improve recognition and management have been evaluated, but have shown no benefit. At least 3 reasons may explain this: education may be insufficient to change practitioner behaviour, study design may have been inadequate to detect true benefit, or the principles on which guidelines are based may be at fault. One reason to suspect the latter is that most of the evidence base comes from secondary care, reflecting a lack of research in primary care. "Sensitivity" of practitioners has been emphasised at the expense of "specificity", which may result in failure to target patients who may benefit most from more intensive management.

The studies by Katzelnick $e t a l$ and Simon $e t$ al report the efficacy and cost effectiveness of a practice based intervention to improve depression management in the managed care context of 3 US HMOs. In a 2 stage screening procedure, patients with consultation rates above the 85 th percentile for $\geqslant 2$ years were identified, and those in whom there was evidence of untreated depressive disorder ( $D S M-I V$ diagnosis, HDRS score $\geqslant 15$ ) were recruited (about $5 \%$ of the total registered population). The study had high completion rates, but only patients enrolled with the HMO for $\geqslant 2$ years were eligible. This limits the replicability and generalisability of the work for other settings (such as UK general practice), biasing against groups such as the long term unemployed or elderly. Half the sample had $\geqslant 1$ comorbid chronic physical illness, presumably accounting in part for their high consultation rates.

Depression management programmes have already been shown to be of benefit for unselected depressed patients in primary care. In this study, programme patients in the DMP had better outcomes than those receiving usual care, with significantly greater reductions in depression scores and higher continued on next page 


\section{A depression management programme increased depression free days and costs in depressed frequent users of general healthcare}

Simon GE, Manning WG, Katzelnick DJ, et al. Cost-effectiveness of systematic depression treatment for high utilizers of general medical care. Arch Gen Psychiatry 2001 Feb;58:181-7.

QUESTION: In depressed patients who are frequent healthcare users, what is the incremental cost effectiveness of a depression management programme (DMP)?

\section{Design}

Cost effectiveness analysis of a cluster randomised (unclear allocation concealment*), partially blinded (telephone assessment)*, controlled trial with 12 months of follow up.

\section{Setting}

3 health maintenance organisations (HMOs) in the US (Wisconsin, Massachusetts, and Washington).

\section{Patients}

407 patients (mean age 45 y, $77 \%$ women) who were frequent users of general medical care $(>85$ th percentile for the number of visits in each of the previous 2 years) and were depressed (Hamilton Depression Rating Scale [HDRS] score $\geqslant 15$ ). Exclusion criteria were active treatment for depression in previous 90 days or contraindications to depression treatment. Analysis included 92\% of patients for healthcare use and $91 \%$ for cost effectiveness.

\section{Intervention}

$\{82\} \dagger$ physician practices were allocated to a DMP $(n=218)$ and $\{81\} \nmid$ were allocated to usual care $(n=189)$. DMP consisted of education and telephone care management, antidepressant treatment for most patients, and psychiatric consultation for non-responders.

\section{Main cost and outcome measures}

The main outcome was number of depression free days (estimated by interpolation). Direct costs were assessed for all services provided or paid for by health plans in 1996
US dollars. Costs for time in treatment were estimated as lost wages. Results were adjusted for age, sex, study site, baseline measures of depression severity and health status, and clustering of patients by physicians.

\section{Main results}

The DMP group had more depression free days than the usual care group (229.3 v $181.9 \mathrm{~d}$; mean adjusted difference 47.4 d, $95 \%$ CI 26.6 to 68.2 d). The table shows the incremental costs of the DMP relative to usual care.

\section{Conclusions}

In depressed patients who are frequent users of general healthcare, a depression management programme was effective for improving clinical outcomes at increased health services cost. Outpatient and inpatient services each cost approximately $\$ 20$ per additional depression free day.

*See glossary.

†Katzelnick DJ, Simon GE, Pearson SD, et al. Randomized trial of a depression management program in high utilizers of medical care. Arch Fam Med 2000;9:345-51.
Source of funding:

Pfizer Pharmaceuticals,

New York, USA.

For correspondence: Dr G E Simon, Center

for Health Studies,

Group Health

Cooperative, 1730

Minor Avenue, Suite

1600, Seattle, WA

98101-1448, USA.

$\mathrm{Fax}+1206287$

2871.

A modified version of this abstract appears in ACP Journal Club.

Incremental cost of a depression management programme relative to usual care

\begin{tabular}{lll} 
Outcomes at 12 months & $\begin{array}{l}\text { Adjusted incremental } \\
\text { cost }(95 \% \text { Cl)‡ }\end{array}$ & $\begin{array}{l}\text { Adjusted cost per } \\
\text { additional depression free } \\
\text { day (CI) }\end{array}$ \\
\hline Outpatient health services & $\$ 1008(534$ to 1383$)$ & $\$ 21.12(10.53$ to 37.61$)$ \\
\hline Plus inpatient services & $\$ 1974(848$ to 3171$)$ & $\$ 41.34(16.04$ to 81.03$)$ \\
\hline Plus time in treatment costs & $\$ 2475(880$ to 4138$)$ & $\$ 51.84(17.37$ to 108.47$)$ \\
\hline
\end{tabular}

fadjusted for age, sex, study site, baseline depression severity, and costs for the 12 months before randomisation.

\section{COMMENTARY — continued from previous page}

quality of life ratings. The authors estimate a number needed to treat of 5 to achieve 1 additional remission, although it is not clear how remission was defined for this calculation. This is a screening study, however, and the "number needed to screen" to achieve 1 additional remission is close to 17 . Consultation rates in the intervention group increased further, while those in the usual care group decreased slightly. It is not possible to identify which component of the programme was of most benefit, but it seems likely on clinical grounds that the initial visit for assessment and initiation of management (which did not occur in the usual care group) would have had considerable impact.

In analysing the cost effectiveness of the programme, the construct of "depression free days" was used. It is important to understand how these were derived. As depression scores were only assessed at 6 weeks and 3,6, and 12 months, most of the data were interpolated. HDRS scores $\leqslant 7$ were taken as "depression free" (score 0 ) while scores $\geqslant 22$ were taken as "fully symptomatic" (score 1). Linear interpolation was used to model recovery, allowing calculation of a number between 0 and 1 for each day, and "depression free days" by dividing the total scores by the number of days in the period between estimates. This is clearly a notional construct, and its name is misleading, because many of the periods will have contained no true depression free days, only partially depression free days.

The costs of the intervention appear high, and there was no evidence of the hoped for "cost offset" effect by reduction in other sources of healthcare cost, perhaps not surprising given the high prevalence of comorbid physical illness. It should be noted that the authors observed that the study was insufficiently powered to compare frequency of inpatient admission, which is costly, and this might have led to failure to detect benefits. The study also only had 1 year of follow up, and benefits may take longer to be detected.

The clinical "bottom line" is that it is possible to identify unmet need and improve outcome in this segment of the primary care population, but substantial additional resources are required. Although these are not out of line with the costs of treating other important conditions, priorities have to be established to permit shifting of existing resources away from other therapeutic areas, or investment of greater resources in this one.

Robert Peveler, MA, DPhil, BM, BCh, FRCPsych University of Southampton and Royal South Hants Hospital, Southampton, UK 